\title{
Differentiation of autonomic reflex control begins with cellular mechanisms at the first synapse within the nucleus tractus solitarius
}

M.C. Andresen,

M.W. Doyle,

T.W. Bailey and

Y.-H. Jin
Department of Physiology and Pharmacology,

Oregon Health and Science University, Portland, OR, USA

\section{Correspondence}

M.C. Andresen

Department of Physiology and

Pharmacology

$O$ regon Health and Science University

Portland, OR 97239-3098

USA

Fax: + 1-503-494-4352

E-mail: andresen@OHSU.edu

This review was based in part on a symposium presentation at the kind invitation of the Brazilian Federation of Societies of Experimental Biology, Salvador, BA, Brazil, August 29-31, 2002

Research supported by the National Institutes of Health (HL-41119 (M.C. Andresen), HL-56460

(M.C. Andresen), HL-70433

(T.W. Bailey)).

The present address of M.W. Doyle is Department of Biology, George Fox University, Newberg, OR 97132-2697, USA.

Received July 24, 2003 Accepted January 21, 2004

\section{Abstract}

Visceral afferents send information via cranial nerves to the nucleus tractus solitarius (NTS). The NTS is the initial step of information processing that culminates in homeostatic reflex responses. Recent evidence suggests that strong afferent synaptic responses in the NTS are most often modulated by depression and this forms a basic principle of central integration of these autonomic pathways. The visceral afferent synapse is uncommonly powerful at the NTS with large unitary response amplitudes and depression rather than facilitation at moderate to high frequencies of activation. Substantial signal depression occurs through multiple mechanisms at this very first brainstem synapse onto second order NTS neurons. This review highlights new approaches to the study of these basic processes featuring patch clamp recordings in NTS brain slices and optical techniques with fluorescent tracers. The vanilloid receptor agonist, capsaicin, distinguishes two classes of second order neurons (capsaicin sensitive or capsaicin resistant) that appear to reflect unmyelinated and myelinated afferent pathways. The differences in cellular properties of these two classes of NTS neurons indicate clear functional differentiation at both the pre- and postsynaptic portions of these first synapses. By virtue of their position at the earliest stage of these pathways, such mechanistic differences probably impart important differentiation in the performance over the entire reflex pathways.
Key words

- Sensory

- Vanilloid

- Glutamate

- Presynaptic modulation

- Autonomic

- Visceral

- Potassium currents

\section{Introduction}

Rapid adjustments of the cardiovascular system depend on neural reflexes and the pathways for these networks are dominated by brainstem neurons $(1,2)$. The effector mechanisms are primarily within the autonomic nervous system and together the re- flexes provide integrated control of multiple organ systems for the maintenance of organism-wide homeostasis. For the most rapid adjustments in hemodynamics (e.g., with postural changes), these reflexes begin with changes in the activity of cardiovascular afferents that in turn serve as inputs to the first central neurons lying within the nucleus trac- 
tus solitarius (NTS). The NTS and these primary visceral afferent synapses thus constitute the first step of information processing. Recent findings suggest that this afferent synapse is the site of a surprising degree of differentiation associated with visceral afferent subtypes. Thus, NTS pathways are distinctly different on the basis of afferent myelination and these differences begin at the afferent terminal (presynaptic characteristics) and are also related to the properties of the NTS neurons receiving those afferents (i.e., the second order NTS neurons). The present review will focus on the basic mechanisms and organization of these differences within the NTS and how such early differentiation in the NTS may alter the simplest views of the brain stem integration. The review will concern, in particular, the ex-

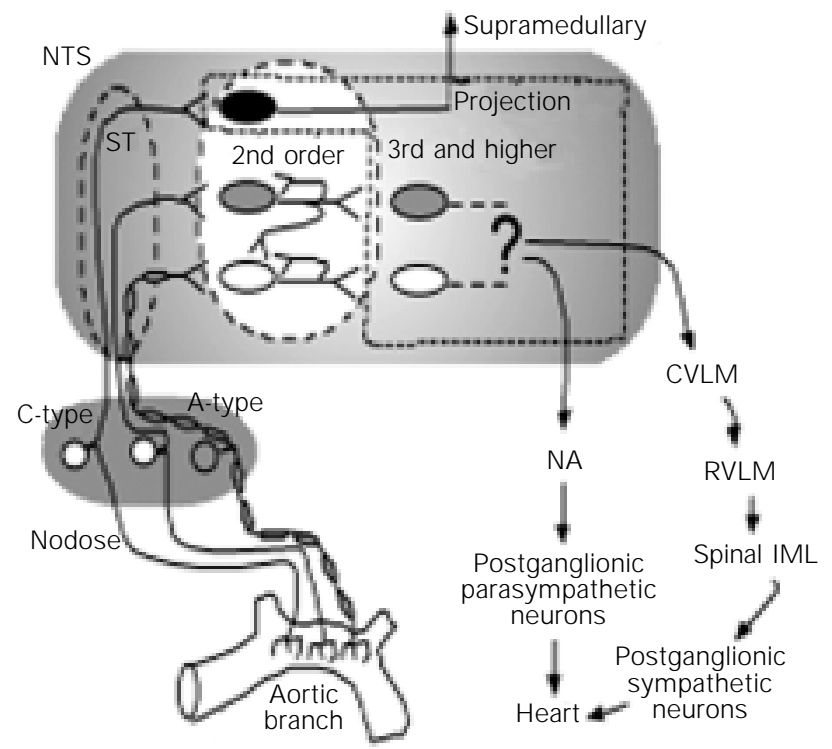

Figure 1. Schematic presentation of the basic organization of brain stem reflex networks for autonomic cardiovascular control. Afferents enter the nucleus tractus solitarius (NTS) to contact second order NTS neurons that have either myelinated, A-type, or unmyelinated, C-type, axons. Second order neurons may contact other neurons within the NTS (i.e., third order interneurons) or send axons out of the NTS to other brain areas (e.g., supramedullary regions such as the hypothalamus). Prominent projections are nucleus ambiguus (NA) and the rostral ventrolateral medulla (RVLM). Neurons in the caudal ventrolateral medula (CVLM) provide feedback to premotor sympathoexcitatory neurons in the RVLM. Preganglionic sympathetic neurons are found in the spinal cord at the intermediolateral cell column (IML). ST = solitary tract. ample of cardiovascular regulation (Figure 1).

\section{Core brainstem circuits for autonomic pathways}

The most basic schemes representing autonomic reflex circuits can be relatively simply constructed as point-to-point sketches of central pathways that connect afferent inputs to efferent autonomic outflows (Figure 1). Lumping each region for the moment as a single functional station, the NTS receives information directly from visceral afferents (e.g., nodose ganglia) and this information ultimately exits the NTS to separate sympathetic and parasympathetic pathways to the heart. The general pattern of central connections for the sympathetic and parasympathetic systems is distinctly different (Figure 1). Data that support such a generalized conceptual diagram derive from multiple experimental sources (e.g., ranging from anatomical point-to-point tracing to functional assays of blood pressure responses to local drug microinjection) (1). Note that, in general, the consensus view represented in this scheme (Figure 1) identifies core paths for autonomic circuits as residing within the brainstem. Clearly, these region-to-region representations of even these core pathways are simplifications that do not incorporate important details of organization (cell to cell contacts or interactions) or information about the cellular mechanisms regulating that pathway. Much of this detail of the mechanisms by which these general areas interact remains to be established. From a circuitry or pathway perspective, fundamental questions of organization for the NTS might include addressing whether second order neurons in the NTS are interneurons (with axons limited to the NTS), projection neurons or a mixture of both (Figure 1). NTS second order neurons are known to follow a loose viscerotopic distribution pattern based on afferent innervation (3). These second order NTS neurons, however, are likely to overlap 
in function so that NTS neurons may contribute to the circuitry of multiple defined major functions (e.g., cardiovascular and respiratory) (3).

One of the structurally simplest autonomic reflex pathways may be the cardiac parasympathetic arc (4). Thus, in concept, this simplest pathway for control of the heart would have only two central neurons, both of which are located in the brainstem. The second order neurons are found in the NTS and the efferent motor neurons in the nucleus ambiguus and/or dorsal motor nucleus of the vagus $(5,6)$. The cardiac preganglionic parasympathetic neurons are diffusely distributed and are intermixed with adjacent neurons projecting elsewhere (6-9). In addition to central "cardiac" neurons, minimal parasympathetic circuits include the primary visceral afferent neurons that feed into the NTS (2) plus postganglionic efferent neurons within the heart (10). Evidence for such a four-neuron full reflex loop includes calculations of reflex latencies (11) and the time course of distributions of retrograde viral tracers (5). Much more complicated and indirect pathways are possible and cardiac parasympathetic control is likely to include additional, more convoluted processing pathways. Nonetheless, the parasympathetic pathways appear distinctly more compact than the sympathetic network (Figure 1). Even at the level of the brainstem, sympathetic pathways appear to be anatomically diffuse and are incompletely understood (see, e.g., Ref. 12). The remainder of this review will focus on the earliest stage of autonomic pathways at NTS second order neurons (shaded box, Figure 1). New details on the nature of processing of afferent inputs to the NTS indicate sharp differentiation well before reflex responses emerge to the broader brainstem.

\section{Baroreceptor reflexes and use-dependent behavior}

Baroreceptor reflexes are particularly in- teresting from a functional perspective because, unlike most somatic reflexes, these circuits are constantly engaged on a second to second basis. Even under "resting" conditions including sleep, baroreceptors are cyclically active with bursts of action potentials during each cardiac cycle (13). One of the enduring mysteries of the NTS, however, has been the paucity of NTS neurons that display cardiac rhythmicity in their own discharge patterns (see, e.g., Refs. 14,15). Clearly, the anesthetics present in these intact animals may alter afferent-NTS coupling and may obscure or distort important aspects of synaptic transmission. However, both cardiac and arterial baroreceptors should convey an intermittent afferent signal that has a strong cardiac synchronicity and interestingly such rhythms are present in sympathetic premotor neurons in the rostral ventrolateral medulla - many neurons removed from the primary afferent inputs (see, e.g., Ref. 16). Baroreceptor reflex responses have long been known to be larger during pressure increases than decreases at the same absolute pressure and, therefore, show substantial hysteresis or directional sensitivity (e.g., 17). Interestingly, under resting conditions, the baroreflex normally operates within the highest sensitivity region (18). Thus, resting values of heart rate and blood pressure reflect ongoing baroreflex actions (sympathoinhibition and parasympathetic tone). These resting values mean that blood pressure excursions normally traverse the midpoint of the reflex performance curve - often described as a sigmoid or logistic relation (19). These characteristics highlight the potential importance of ongoing activity, the direction of activity changes and frequencydependent processes in normal function of this reflex pathway. Experimental evidence reinforces this activity dependence since the baroreflex is "reset" by changes in activity (20). This use-dependence extends to nonpathological chronic conditions since baroreflexes observed in humans and in experi- 
mental animals are substantially modified by periods of sustained hemodynamic alterations such as bed rest or zero gravity (21). Little is known about the mechanisms responsible or site(s) of alteration that such plasticity suggests. Since the most basic core pathways may involve relatively few neurons and synapses, understanding vagal heart rate control, for example, may focus on a surprisingly limited number of potential sites of action. The second order NTS neurons represent an interesting common "gateway" for these reflex pathways - no matter whether these paths contain very few neurons and are relatively direct or are part of more convoluted paths (Figure 1).

\section{The case for the strong synapse}

The synapses from cranial visceral afferents within the medial portions of the NTS are unusual since they contact these second order neurons close to the soma and the resulting unitary synaptic currents are uncommonly large with low failure rates $(22,23)$. Together, these characteristics create a strong synapse and a particularly high fidelity of transmission of excitation for that first afferent action potential through the next neuron in the pathway. NTS afferent transmission with large unitary responses from relatively few contacts is quite different from the classical view of central synaptic transmission as resulting from integration of large numbers of individually weak events (24).

The precise mechanisms that underlie the transfer and transformation of information from visceral afferent into discharge of NTS second order neurons are often difficult to resolve in vivo for many technical reasons. To better examine these mechanisms, the in vitro patch clamp approach has important advantages in experimental control and much improved resolution (22). Horizontal slices containing the NTS effectively isolate afferent solitary tract (ST) axons and pro- vide for selective electrical activation of ST axons to carefully examine synaptic timing. The large amplitude of the ST-evoked synaptic currents (commonly $300-600 \mathrm{pA}$ ) is a key characteristic of visceral afferents with important implications for synaptic integration (22). Additional hallmarks include the substantial depression of ST transmission at physiologically important frequencies (above $5 \mathrm{~Hz}$ ) and, more controversially, the reliance of second order neurons on non-NMDA glutamate receptors $(22,25)$ (for more on this, see below). Since synaptic transmission operates close to maximum effectiveness (e.g., very high glutamate release probability), inhibitory mechanisms and/or depression of glutamate release are a critical target of modulation of this afferent synapse.

In vitro experiments arose from a rich history of work using varied approaches devoted to discerning the neurotransmitter(s) responsible for transmission of afferent excitation at the NTS level. Glutamate is clearly the primary neurotransmitter (1). The earliest NTS experiments elicited several interesting and controversial puzzles about the broader role of glutamate and specific receptors in the NTS. Early experiments used then newly developed selective drugs and suggested that both non-NMDA and NMDA glutamate receptors participate in CNS responses such as the baroreceptor reflex. Microinjection studies lead the way by placing glutamate selective agents directly into the NTS region (26). Subtype specific ionotropic glutamate receptor antagonists blocked baroreflex responses but paradoxically not responses to injected glutamate (27). Various in vivo and cellular NTS studies followed and confirmed this broad, ionotropic glutamatergic receptor participation (28-33). Unanswered questions remained, however, as to what was the site of action of these drugs and whether all neurons in the NTS were the same.

The issue of the heterogeneity of NTS neurons is only now beginning to yield to 
experimental work. For example, evidence from intracellular recordings in horizontal slices $(22,34)$ does not support a substantial or direct role for NMDA receptors in ST afferent transmission. In most identified second order NTS neurons, glutamate - whether ST released or exogenous - fails to activate NMDA receptors even in the absence of extracellular $\mathrm{Mg}^{2+}(22,34,35)$. The exception is a small population of second order NTS neurons with apparently myelinated ST afferent innervation (36). Methods of approach are critical considerations for interpreting results and, clearly, alternative explanations are needed to account for NMDA receptor responses in the NTS. For example, in transverse NTS slice preparations, monosynaptic inhibitory synaptic responses (GABAa mediated) are commonly found with electrical shocks in the vicinity of the ST (see, e.g., Ref. 37). The close proximity of the stimulating electrode appears to recruit local inhibitory interneurons and thus non-ST responses are clearly evident in such circumstances. Interestingly, NTS interneurons are activated by powerful NMDA synaptic components (38) and thus are pharmacological targets during microinjection that are intermixed with second order neurons. Thus, the net effect on blood pressure or reflex function reflects a summation of all direct and indirect actions at multiple receptor subtypes and locations. Understanding the contributions of this heterogeneity will require a concerted effort and focused experimental design.

\section{Heterogeneity in afferent neurons to the NTS}

Second order NTS neurons are, by definition, driven by ST excitatory synaptic inputs from visceral primary afferents arriving through the cranial nerves. The cellular properties of visceral afferent neurons such as those in the nodose ganglion are highly varied (3). Relatively direct access to the pe- ripheral cell bodies and sensory terminals shows that mechanoreception and chemoreception are dominant sensory modalities among these cranial visceral primary afferents $(3,39)$. Cardiovascular afferents including arterial baroreceptors provide an interesting example since their physiological activation is now being translated into cellular mechanisms in increasing detail $(40,41)$. A dominating, if under-appreciated, facet of the characteristics of cranial visceral afferent neurons, however, is their intrinsic variability (42-44). Cranial visceral afferent neurons belong to two fundamental subdivisions (Figure 2), those with myelinated axons (A-type) and those with unmyelinated axons (C-type). A-type baroreceptors tend to have very regular discharge patterns and encode arterial pressure with great fidelity while Ctype baroreceptors tend to have lower, often

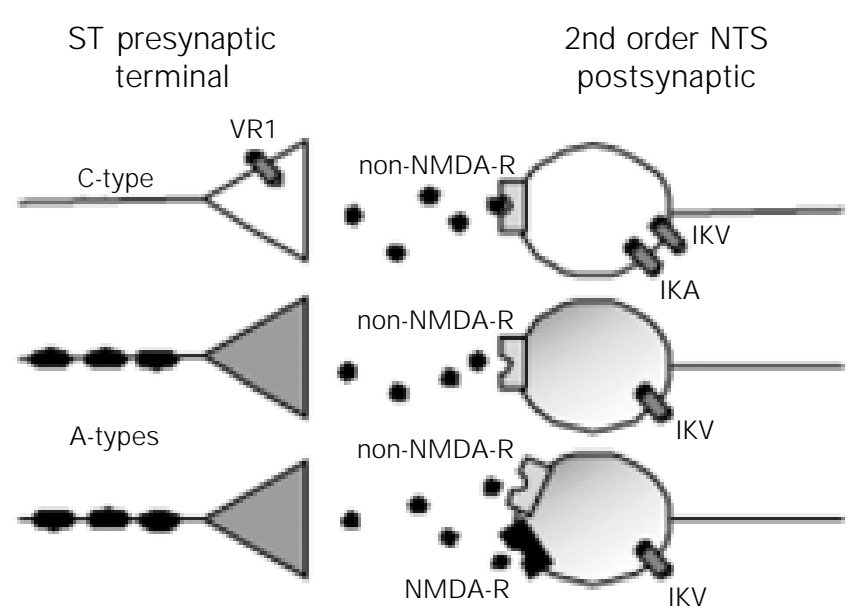

Figure 2. Solitary tract (ST) afferents fall into two broad classes based on the presence of the vanilloid receptor (VR1). Capsaicin-sensitive afferent axons are considered to be unmyelinated, C-type. A-type, myelinated ST axons as well as postsynaptic neurons are devoid of VR1. The anesthetic ketamine blocks NMDA receptors of a subset of A-type ST innervated neurons. Thus, presynaptic A- and C-type ST afferent terminals are highly differentiated on the basis of ligand- and voltage-dependent ion channel expression. Differential expression of pre- and postsynaptic effector proteins is likely to extend to particular ion channels as well as receptors (ionotropic and metabotropic). This offers multiple sites of potential action even for a single transmitter. These differences may provide the mechanistic basis for differential processing unique to each pathway. IKA = A-type potassium current; $\mathrm{IKV}=$ voltage-dependent potassium current; NMDA-R = N-methyl-Daspartic acid postsynaptic receptors; NTS = nucleus tractus solitarius. 
sporadic discharge patterns that are only roughly proportional to pressure (45). Observations of the reflex responses evoked by selective activation of these neuron classes suggest that these two pools of sensory afferent neurons give rise to reflexes with interesting differences in overall performance (46-48). For example, substantial cardiovascular reflex responses are evoked by as few as one action potential per second for C-type aortic baroreceptors, whereas reflex responses emerge from A-type baroreceptor activation only at 10 action potentials per second or higher $(46,47)$. A-type and C-type neurons differ in the properties at the cell soma (49). However, new results suggest that important basic cellular differences also separate the central terminations into pharmacologically and functionally distinct groups (Figure 2). Together, this heterogeneity importantly shapes afferent central transmission.

A hallmark of the neurons within the NTS is broad cellular heterogeneity. Clearly, only a portion of the neurons within the NTS act as second order neurons - that is they receive afferent ST synaptic contacts. Other neurons in the NTS serve as either interneurons with contacts contained within the NTS or as projection neurons that send axons out of the NTS to other CNS areas. Anatomic studies have provided information about the wide spectrum of point-to-point connections between NTS and other CNS structures $(3,50)$ although very little specific information about the nature of these interconnections is established. Anatomical subdivisions of the NTS have been described using histological and anatomical criteria by different investigators $(51,52)$, but it is not clear what are the broader functional or mechanistic ramifications of such NTS diversity. Neurotransmitters and their receptors range tremendously with topographically varied expression within the NTS, but most classification groups are also without defined function or target $(2,53)$. Neurons have been divided into different classes of discharge patterns or ion channel expression in both caudal and rostral NTS and these key characteristics are linked to potassium channels $(54,55)$. Thus, a major challenge in this area of the brainstem is to link these cellular characteristics with function and/or identified pathways.

\section{Cellular basis of NTS neurotransmission}

The work summarized here considers the idea that, despite basic similarities of glutamatergic transmission, other prominent differences in pre- and postsynaptic neurons shape an important differentiation of second order NTS neurons. We began with the premise that peripheral cellular differences in cranial afferent neurons (e.g., at the nodose ganglion) are present in the central, presynaptic terminals of these same sensory neurons. The expectation (Figure 2) is that pathways through the NTS would be divided on the basis of afferent class $(34,36,56)$.

Second order neurons in the NTS were selected by both electrophysiological and/or anatomical criteria, e.g., visualization of sensory boutons on the NTS neuron soma (22). ST shocks activated excitatory postsynaptic currents (EPSCs) with a range of latencies (22). Among the neurons recorded from such slices, a subset possesses ST-evoked EPSCs with nearly invariant latencies (low jitter) reflecting monosynaptic ST contacts identifying them as second order NTS neurons (22). These fast EPSCs activate non-NMDA postsynaptic receptors (Figure 2) in nearly all cases, confirming that the primary transmitter is glutamate (Figure 2).

We have recently developed a new strategy for identification of NTS second order neurons innervated by unmyelinated, C-type afferents. The vanilloid receptor VR1 is selectively expressed in a subset of spinal sensory neurons - typically those with unmyelinated or lightly myelinated axons in dorsal root ganglion neurons $(57,58)$. VR1 is coupled to an ion channel that fluxes cations 
including a high conductance for calcium ions $(57,58)$. Capsaicin (CAP), the active ingredient in pungent peppers, is a selective ligand for VR1. CAP painted onto the aortic depressor nerve completely and selectively blocks the conduction of C-type axons and their cardiovascular reflex responses (46). Thus, we decided to test this mode of chemical modulation on brain stem slices containing the NTS. In close correspondence to the peripheral action of CAP, we found two types of responses in second order NTS neurons. Although both types of neurons responded to ST stimulation with very similar, low jitter EPSCs ( $<150$ microsec jitter), the synaptic transmission to one set of neurons (CAP-sensitive) was blocked by CAP and the other neurons were unaffected by CAP (CAP-resistant). CAP applied to such slices triggered a burst of spontaneous synaptic currents that declined in the continued presence of CAP (34). CAP responses were prevented by application of selective nonNMDA receptor antagonists and unaltered by application of NMDA receptor antagonists. CAP appears to trigger a massive release of glutamate containing synaptic vesicles. Thus, pharmacologically, CAP responses mimic ST afferent synaptic activation. The blockade of ST synaptic transmission by CAP appears to be a presynaptic action since postsynaptic glutamate receptors remain responsive to exogenous agonists during CAP block of electrically triggered ST synaptic transmission. Together, such results are consistent with specific CAP actions on VR1 receptors present only on the terminals of C-type ST afferent axons (Figure 2). Interestingly, no partial blockades were found - an observation that suggests that A-type and C-type afferent axons do not converge on the same second order neuron in the NTS. The mechanism of C-type ST axon blockade is unclear. Intensive activation of C-type axons might deplete the neurotransmitter. Depolarization block of presynaptic terminals could contribute to synaptic blockade during CAP. Although multiple mechanisms might contribute, the time course of onset of inhibition and its very slow reversal are consistent with depletion of synaptic vesicles from presynaptic terminals. These experiments provide one example of what is likely to be a broad presynaptic heterogeneity of primary afferent innervation of the NTS (Figure 2).

Surprisingly, the differences in primary afferent terminals lead to the identification of differences in postsynaptic properties of second order NTS neurons. CAP-sensitive and CAP-resistant neurons have significantly different voltage-dependent potassium currents. Two-step voltage clamp studies revealed that CAP-sensitive and CAP-resistant afferent subtypes are uniquely associated with different types of medial NTS neurons. CAP-sensitive neurons possess a much more prominent A-type potassium current (IKA) that is sensitive to millimolar concentrations of 4-aminopyridine, while all second order NTS neurons have a similar expression of voltage-dependent potassium current, IKV (Figure 2). These prominent differences in postsynaptic potassium channels alter the translation of afferent synaptic inputs into second order neuron discharge (56). The differences in potassium channels are particularly interesting since they correspond to the known differences between Atype and C-type nodose neurons. The early transient IKA can act as a significant brake on the translation of excitatory synaptic potentials into action potentials in NTS second order neurons (56). Since NTS neurons with prominent IKA are contacted predominantly by C-type afferents, this may be a mechanism that contributes to the low frequency bias of these C-type pathways. Such potassium currents represent different targets for neurotransmitters (e.g., Refs. 59,60). Accessory neurotransmitters such as peptides are closely associated with C-type afferents (49). This suggests a number of interesting potential future avenues of research to discover 
whether release characteristics of neurotransmission are matched to specific postsynaptic properties and how those impact efficacy of overall translation of input to output. Given the differences in neurotransmitters expressed by sensory afferents, the differences in postsynaptic NTS neurons could represent an important component mechanism of differential afferent processing. For cardiovascular regulation, future work should be targeted specifically toward aortic baroreceptor terminals on NTS neurons (22) to see if this subtype matching exists in cardiovascular pathways.

In summary, detailed studies of synaptic transmission and ionic currents in second order NTS neurons reveal an unexpected differentiation of reflex pathways at these first CNS neurons. Such findings have strong implications for our understanding of the determinants of the overall reflex performance. Not only are the afferent discharge patterns different in myelinated and unmyelinated afferents, the reception of the released neurotransmitter at the second order neurons is shaped in fundamental ways by the differences in ion channel expression of the central neurons. This early segregation of pathways produces substantially different reflex paths with performance characteristics that are tuned differentially to these distinct afferent inputs. The differential expression of VR1 across presynaptic afferent ST axons is likely to presage other presynaptic patterns that hold the prospect of chemical coding or selective interventional strategies that affect subsets of reflex pathways. Many details relating to afferent modality (e.g., cardiovascular, respiratory or gastrointestinal) remain largely unknown so that much work remains ahead before the potential impact of these heterogeneities can be fully appreciated or exploited.

\section{References}

1. Pilowsky PM \& Goodchild AK (2002). Baroreceptor reflex pathways and neurotransmitters: 10 years on. J ournal of Hypertension, 20: 1675-1688.

2. Andresen MC \& Kunze DL (1994). Nucleus tractus solitarius: gateway to neural circulatory control. Annual Review of Physiology, 56: 93-116.

3. Loewy AD (1990). Central autonomic pathways. In: Loewy AD \& Spyer KM (Editors), Central Regulation of Autonomic Functions. Oxford University Press, New York, 88-103.

4. Standish A, Enquist LW \& Schwaber J S (1994). Innervation of the heart and its central medullary origin defined by viral tracing. Science, 263: 232-234.

5. Standish A, Enquist LW, Escardo J A \& Schwaber J S (1995). Central neuronal circuit innervating the rat heart defined by transneuronal transport of pseudorabies virus. J oumal of Neuroscience, 15: 19982012.

6. Irnaten M, Neff RA, Wang J, Loewy AD, Mettenleiter TC \& Mendelowitz D (2001). Activity of cardiorespiratory networks revealed by transsynaptic virus expressing GFP. J oumal of Neurophysiology, 85: 435-438.

7. Mendelowitz D (2000). Superior laryngeal neurons directly excite cardiac vagal neurons within the nucleus ambiguus. Brain Research Bulletin, 51: 135-138.

8. Mendelowitz D (1998). Nicotine excites cardiac vagal neurons via three sites of action. Clinical and Experimental Pharmacology and Physiology, 25: 453-456.
9. Mendelowitz D (1999). Advances in parasympathetic control of heart rate and cardiac function. News in Physiological Sciences, 14: 155-161.

10. Thompson GW, Collier K, Ardell J L, Kember G \& Armour J A (2000). Functional interdependence of neurons in a single canine intrinsic cardiac ganglionated plexus. J ournal of Physiology, 528: 561-571.

11. Kunze DL (1972). Reflex discharge pattems of cardiac vagal efferent fibres. J oumal of Physiology, 222: 1-15.

12. Morrison SF (2001). Differential control of sympathetic outflow. American J ournal of Physiology, 281: R683-R698.

13. Thoren PN \& J ones J (1977). Characteristics of aortic baroreceptor C-fibers in the rabbit. Acta Physiologica Scandinavica, 99: 448-456.

14. Hayward LF \& Felder RB (1995). Cardiac rhythmicity among NTS neurons and its relationship to sympathetic outflow in rabbits. American J ournal of Physiology, 269: H923-H933.

15. Lambertz M, Kluge W \& Langhorst P (1993). Discharge pattem of neurons in the nucleus tractus solitarii (NTS): its cardiac rhythm is modulated by firing rate of the neurons. J ournal of the Autonomic Nervous System, 44: 137-150.

16. Brown DL \& Guyenet PG (1985). Electrophysiological study of cardiovascular neurons in the rostral ventrolateral medulla in rats. Circulation Research, 56: 359-369.

17. Brunner MJ , Sussman MS, Greene AS, Kallman CH \& Shoukas AA (1982). Carotid sinus baroreceptor reflex control of respiration. Circulation Research, 51: 624-636.

18. Dorward PK, Andresen MC, Burke SL, Oliver J R \& Komer PI (1982). 
Rapid resetting of the aortic baroreceptors in the rabbit and its implications for short-term and longer term reflex control. Circulation Research, 50: 428-439.

19. Ricketts J H \& Head GA (1999). A five-parameter logistic equation for investigating asymmetry of curvature in baroreflex studies. American J ournal of Physiology, 277: R441-R454.

20. Kunze DL (1986). Acute resetting of baroreceptor reflex in rabbits: a central component. American J ournal of Physiology, 250: H866$\mathrm{H} 870$.

21. Eckberg DL \& Fritsch J M (1992). Influence of ten-day head-down bedrest on human carotid baroreceptor-cardiac reflex function. Acta Physiologica Scandinavica Supplement, 604: 69-76.

22. Doyle MW \& Andresen MC (2001). Reliability of monosynaptic transmission in brain stem neurons in vitro. J ournal of Neurophysiology, 85: 2213-2223.

23. Andresen MC \& Yang M (1995). Dynamics of sensory afferent synaptic transmission in aortic baroreceptor regions of nucleus tractus solitarius. J ournal of Neurophysiology, 74: 1518-1528.

24. Trussell LO (1999). Synaptic mechanisms for coding timing in auditory neurons. Annual Review of Physiology, 61: 477-496.

25. Andresen MC, Doyle MW, J in Y-H \& Bailey TW (2001). Cellular mechanisms of baroreceptor integration at the nucleus tractus solitarius. Annals of the New York Academy of Sciences, 940: 132-141.

26. Guyenet PG, Filtz TM \& Donaldson SR (1987). Role of excitatory amino acids in rat vagal and sympathetic baroreflexes. Brain Research, 407: 272-284.

27. Leone C \& Gordon FJ (1989). Is L-glutamate a neurotransmitter of baroreceptor information in the nucleus of tractus solitarius? J ournal of Pharmacological and Experimental Therapeutics, 250: 953962.

28. Drewe J A, Miles R \& Kunze DL (1990). Excitatory amino acid receptors of guinea pig medial nucleus tractus solitarius neurons. American J ournal of Physiology, 259: H1389-H1395.

29. Miller BD \& Felder RB (1988). Excitatory amino acid receptors intrinsic to synaptic transmission in nucleus tractus solitarii. Brain Research, 456: 333-343.

30. Seagard J L, Dean C \& Hopp FA (2003). Activity-dependent role of NMDA receptors in transmission of cardiac mechanoreceptor input to the NTS. American J oumal of Physiology, 284: H884-H891.

31. Zhang J \& Mifflin SW (1998). Differential roles for NMDA and nonNMDA receptor subtypes in baroreceptor afferent integration in the nucleus of the solitary tract of the rat. J oumal of Physiology, 511: 733-745.

32. Zhang W \& Mifflin SW (1995). Excitatory amino-acid receptors contribute to carotid sinus and vagus nerve evoked excitation of neurons in the nucleus of the tractus solitarius. J ournal of the Autonomic Nervous System, 55: 50-56.

33. Machado BH \& Bonagamba LGH (1992). Microinjection of L-glutamate into the nucleus tractus solitarii increases arterial pressure in conscious rats. Brain Research, 576: 131-138.

34. Doyle MW, Bailey TW, J in Y-H \& Andresen MC (2002). Vanilloid receptors presynaptically modulate visceral afferent synaptic transmission in nucleus tractus solitarius. J oumal of Neuroscience, 22: 8222-8229.

35. Andresen MC \& Yang M (1990). Non-NMDA receptors mediate sensory afferent synaptic transmission in medial nucleus tractus solitarius. American J ournal of Physiology, 259: H1307-H1311.

36. J in Y-H, Bailey TW, Doyle MW, Li BY, Chang KSK, Schild J H, Mendelowitz D \& Andresen MC (2003). Ketamine differentially blocks sensory afferent synaptic transmission in medial nucleus tractus solitarius (mNTS). Anesthesiology, 98: 121-132.
37. Glaum SR, Brooks PA, Spyer KM \& Miller RJ (1992). 5-Hydroxytryptamine-3 receptors modulate synaptic activity in the rat nucleus tractus solitarius in vitro. Brain Research, 589: 62-68.

38. Titz S \& Keller BU (1997). Rapidly deactivating AMPA receptors determine excitatory synaptic transmission to interneurons in the nucleus tractus solitarius from rat. J oumal of Neurophysiology, 78: 82-91.

39. J änig W \& MCLachlan EM (1992). Characteristics of function-specific pathways in the sympathetic nervous system. Trends in Neurosciences, 15: 475-481.

40. Chapleau MW, Li Z, Meyrelles SS, Ma X \& Abboud FM (2001). Mechanisms determining sensitivity of baroreceptor afferents in health and disease. Annals of the New York Academy of Sciences, 940: 1-19.

41. Hay M, Hoang CJ \& Pamidimukkala J (2001). Cellular mechanisms regulating synaptic vesicle exocytosis and endocytosis in aortic baroreceptor neurons. Annals of the New York Academy of Sciences, 940: 119-131.

42. Schild J H, Clark J W, Hay M, Mendelowitz D, Andresen MC \& Kunze DL (1994). A- and C-type nodose sensory neurons: Model interpretations of dynamic discharge characteristics. J oumal of Neurophysiology, 71: 2338-2358.

43. Schild J H \& Kunze DL (1997). Experimental and modeling study of $\mathrm{Na}^{+}$current heterogeneity in rat nodose neurons and its impact on neuronal discharge. J ournal of Neurophysiology, 78: 3198-3209.

44. Seagard J L, Van Brederode J FM, Dean C, Hopp FA, Gallenberg LA \& Kampine JP (1990). Firing characteristics of single-fiber carotid sinus baroreceptors. Circulation Research, 66: 1499-1509.

45. Kunze DL \& Andresen MC (1991). Arterial baroreceptors: Excitation and modulation. In: Zucker IH \& Gilmore J P (Editors), Reflex Control of the Circulation. CRC Press, Boca Raton, FL, USA, 141-166.

46. Fan W \& Andresen MC (1998). Differential frequency-dependent reflex integration of myelinated and nonmyelinated rat aortic baroreceptors. American J ournal of Physiology, 275: H632-H640.

47. Fan W, Schild JH \& Andresen MC (1999). Graded and dynamic reflex summation of myelinated and unmyelinated rat aortic baroreceptors. American J ournal of Physiology, 277: R748-R756.

48. Seagard J L, Hopp FA, Drummond HA \& Van Wynsberghe DM (1993). Selective contribution of two types of carotid sinus baroreceptors to the control of blood pressure. Circulation Research, 72: 1011-1022.

49. Lawson SN (1992). Morphological and biochemical cell types of sensory neurons. In: Scott SA (Editor), Sensory Neurons: Diversity, Development, and Plasticity. Oxford University Press, New York, 27-59.

50. Spyer KM (1990). The central nervous organization of reflex circulatory control. In: Loewy AD \& Spyer KM (Editors), Central Regulation of Autonomic Functions. Oxford University Press, New York, 168188.

51. Kalia M \& Sullivan J M (1982). Brainstem projections of sensory and motor components of the vagus nerve in the rat. J ournal of Comparative Neurology, 211: 248-264.

52. Ruggiero DA, Pickel VM, Milner TA, Anwar M, Otake K, Mtui EP \& Park D (1994). Viscerosensory processing in nucleus tractus solitarii: structural and neurochemical substrates. In: Barraco RA (Editor), Nucleus of the Solitary Tract. CRC Press, Boca Raton, FL, USA, 334.

53. Lawrence AJ \& J arrott B (1996). Neurochemical modulation of cardiovascular control in the nucleus tractus solitarius. Progress in Neurobiology, 48: 21-53.

54. Butcher J W \& Paton J FR (1998). $\mathrm{K}^{+}$channel blockade in the NTS 
alters efficacy of two cardiorespiratory reflexes in vivo. American J oumal of Physiology, 274: R677-R685.

55. Dekin MS \& Getting PA (1984). Firing pattem of neurons in the nucleus tractus solitarius: modulation by membrane hyperpolarization. Brain Research, 324: 180-184.

56. Bailey TW, J in Y-H, Doyle MW \& Andresen MC (2002). Vanilloid sensitive afferents activate neurons with prominent A-type potassium currents in nucleus tractus solitarius. J ournal of Neuroscience, 22: 8230-8237.

57. Caterina MJ \& J ulius D (2001). The vanilloid receptor: a molecular gateway to the pain pathway. Annual Review of Neuroscience, 24:
487-517.

58. Szallasi A \& Blumberg PM (1999). Vanilloid (Capsaicin) receptors and mechanisms. Pharmacological Reviews, 51: 159-212.

59. Oz M, Kolaj M \& Renaud LP (2001). Electrophysiological evidence for vasopressin $\mathrm{V}(1)$ receptors on neonatal motoneurons, premotor and other ventral horn neurons. J ournal of Neurophysiology, 86: $1202-1210$.

60. Zona C, Farini D, Palma E \& Eusebi F (1991). Modulation of voltageactivated channels by calcitonin gene-related peptide in cultured rat neurones. J ournal of Physiology, 433: 631-643. 